\title{
Arterial Steal to the Penumbra Area in Patients with Acute MCA Occlusion: A Quantitative Angiographic Analysis
}

\author{
René van den Berg, $\mathrm{PhD}^{1}$, Jenna J. Wildeman, $\mathrm{MSc}^{2}$, Olvert A. Berkhemer, $\mathrm{PhD}^{1}$, \\ Rogier V. Immink, $\mathrm{PhD}^{3}$, Henk A. Marquering, $\mathrm{PhD}^{1,4}$, Charles B.L.M. Majoie, $\mathrm{PhD}^{1}$, \\ Dagmar Verbaan, $\mathrm{PhD}^{5}$, Ed T. van Bavel, $\mathrm{PhD}^{4}$ \\ ${ }^{1}$ Department of Radiology and Nuclear Medicine, Amsterdam University Medical Centers (AMC), Amsterdam, The Netherlands \\ ${ }^{2}$ Department of Biomedical Sciences, VU University Amsterdam, Amsterdam, The Netherlands \\ ${ }^{3}$ Department of Anesthesiology, Amsterdam University Medical Centers (AMC), Amsterdam, The Netherlands \\ ${ }^{4}$ Department of Biomedical Engineering and Physics, Amsterdam University Medical Centers (AMC), Amsterdam, The Netherlands \\ ${ }^{5}$ Department of Neurosurgery, Amsterdam University Medical Centers (AMC), Amsterdam, The Netherlands
}

Purpose: In acute middle cerebral artery (MCA) occlusion, collateral vessels provide retrograde supply to the occluded territory. We hypothesized that such collateral flow reduces perfusion of the non-occluded donor region (steal effect).

Materials and Methods: Patients with an MCA occlusion with opacification of both ipsi- and contralateral anterior cerebral arteries (ACA) on angiography prior to endovascular treatment were selected. Arteriovenous transit time (AVTT) for both ACA territories was compared for different grades of collateral supply to the MCA territory. In addition, the influence of diabetes and hypertension was analyzed. After successful revascularization, AVTT was re-assessed to determine reversibility.

Results: Forty-one patients were analyzed. An AVTT of 8.6 seconds (standard deviation [SD] 2.4 seconds) was seen in the ACA territory of the affected hemisphere in comparison to 6.6 seconds (SD 2.1 seconds) for the contralateral side $(P<0.001)$. A more prolonged (but not significant) AVTT was seen in cases with a higher collateral grade. No difference in AVTT was seen in patients with diabetes or hypertension. After successful MCA revascularization, AVTT delay was 7.4 seconds (SD 2.1 seconds).

Conclusion: A cerebral steal effect occurs in patients with an acute MCA occlusion, probably related to augmented flow to the penumbra area.

Key Words: Acute stroke; Arterial occlusive diseases; Collateral circulation; Cerebrovascular circulation; Cerebral angiography

\section{INTRODUCTION}

With the recent positive trials on endovascular treatment (EVT) to re-open middle cerebral artery (MCA) occlusions, digital subtraction angiography (DSA) is now used in the acute setting for interventional guidance and localization of the exact MCA occlusion. ${ }^{1}$ In addition, the temporal resolution of DSA enables depiction of the collateral flow from the anterior and posterior cerebral arteries, through the leptomeningeal anastomosis at the watershed areas towards the occluded MCA territory. ${ }^{2}$ The presence of collateral arteries supplying

\section{Correspondence to: René van den Berg, PhD \\ Department of Radiology and Nucle- ar Medicine, Amsterdam University Medical Centers (AMC), Meibergdreef 9, 1105 AZ, Amsterdam, The Nether- lands \\ Tel:+31-20-7324352 \\ Fax: +31-20-5669119 \\ E-mail: r.vdberg@amsterdamumc.nl}

Received: July 18, 2020

Revised: September 11, 2020

Accepted: September 30, 2020
Copyright $\odot 2020$ Korean Society of Interventional Neuroradiology

This is an Open Access article distributed under the terms of the Creative Commons Attribution Non-Commercial License (http://creativecommons.org/licenses/by-nc/4.0) which permits unrestricted non-commercial use, distribution, and reproduction in any medium, provided the original work is properly cited. 
the occluded MCA territory is associated with an improved clinical outcome. ${ }^{3,4}$ The exact mechanism of opening of the leptomeningeal arteries during acute large vessel occlusion and its place in neurovascular regulation mechanisms is up to now unclear. Transcranial Doppler studies on dynamic cerebral autoregulation have been performed in patients with large MCA territory infarcts and have shown unilateral and bilateral impairment of cerebral autoregulation. ${ }^{5,6}$ The high temporal resolution of DSA allows a better depiction of the supply to the collateral network from the anterior cerebral artery (ACA) territory or the posterior cerebral artery territory. DSA also allows estimation of perfusion of the non-occluded donor region, based on estimation of arteriovenous contrast transit time from arterial inflow in the ACA to venous drainage.

In this study we determined the changes in perfusion in the ACA territory in the affected versus unaffected hemisphere in acute MCA occlusion. Furthermore, we related these changes to collateral flow to the occluded MCA territory from the ACA territory.

\section{MATERIALS AND METHODS}

A retrospective analysis was performed on a large single center prospective cohort of 364 patients (starting May 2011 and ended at June 1st 2017), presenting with an acute hemispheric stroke, due to a proximal MCA occlusion proven on computed tomography (CT) angiography, which was suitable for EVT according to the original Multicenter Randomized Clinical Trial of Endovascular Treatment for Acute Ischemic Stroke in the Netherlands (MR CLEAN) inclusion criteria. ${ }^{7}$ From this cohort, only those patients who showed contrast filling of both the ipsi- and contralateral ACA on DSA were included. During EVT, single catheterization is performed of the internal carotid artery (ICA) supplying the affected hemisphere. To assess the flow in both ACA territories, the anatomical prerequisite is a patent anterior communicating artery (ACOM), allowing contrast passage from the catheterized ICA through the first segment of the ACA to the contralateral distal segments of the ACA.

Patients were selected from the local MR CLEAN and the MR CLEAN Registry database for this post-hoc analysis. A central medical ethics committee granted permission to carry out the original study and additional post-hoc analyses, therefore no additional local approval was required to carry out this study.

\section{Imaging assessment}

All patients were studied on biplane digital subtraction angiographic equipment (Philips Allura Xper FD20/20 biplane neuro X-ray system; Philips Healthcare, Best, The Netherlands). Contrast material (lodixanol 320) was administered with hand injections through the ICA with postero-anterior and lateral acquired images from the early arterial to the late venous phase. A standard triple phase protocol was used with a frame rate of three per second for the first four seconds, one frame per second for six seconds, and finally one frame per two seconds for the final (venous) phase until opacification of the jugular vein is seen. This allowed reliable temporal analysis of the arterial and venous phase and assessment of the leptomeningeal collateral circulation. Collateral circulation was graded using standard anatomical landmarks in which the MCA is subdivided in four segments, the horizontal M1 segment, the ascending insular $M 2$, the descending opercular M3 segment, and the cortical M4 segment. The AVTT was defined as the time interval between the first opacification of the distal ICA and the earliest opacification of the draining veins of the local ACA territory. AVTT was measured for both the affected (side of MCA occlusion) and unaffected hemisphere (Fig. 1). Additional subgroup analysis was performed for the different collateral grades. Immediately after successful revascularization of the MCA a control DSA was performed allowing repeat measurement of the AVTT to record possible changes. Revascularization was graded, using the modified Thrombolysis in Cerebral Infarction ( $\mathrm{TICl}$ ) score, which ranges from 0 (no reperfusion) to 3 (complete reperfusion). ${ }^{8}$ Presence of a residual stenosis of the MCA after successful revascularization was determined in patients with successful revascularization. Age, hypertension, diabetes mellitus, and presence of a significant carotid stenosis were extracted for additional subgroup analysis.

\section{Statistical analysis}

Statistical analysis was performed using SPSS 23.4 (IBM Co., Armonk, NY, USA). First, data was checked for a normal distribution of AVTT using the Shapiro-Wilk test ( $>0.9$ is considered as normally distributed). Since the distribution of AVTT of the unaffected side was not normally distributed, the Wilcoxon signed rank test (as both measurements were from the same DSA from the same patient) was used to test for significant differences in AVTT between the affected and unaffected hemisphere. Since the difference in AVTT between the affected and unaffected side was normally distributed, 

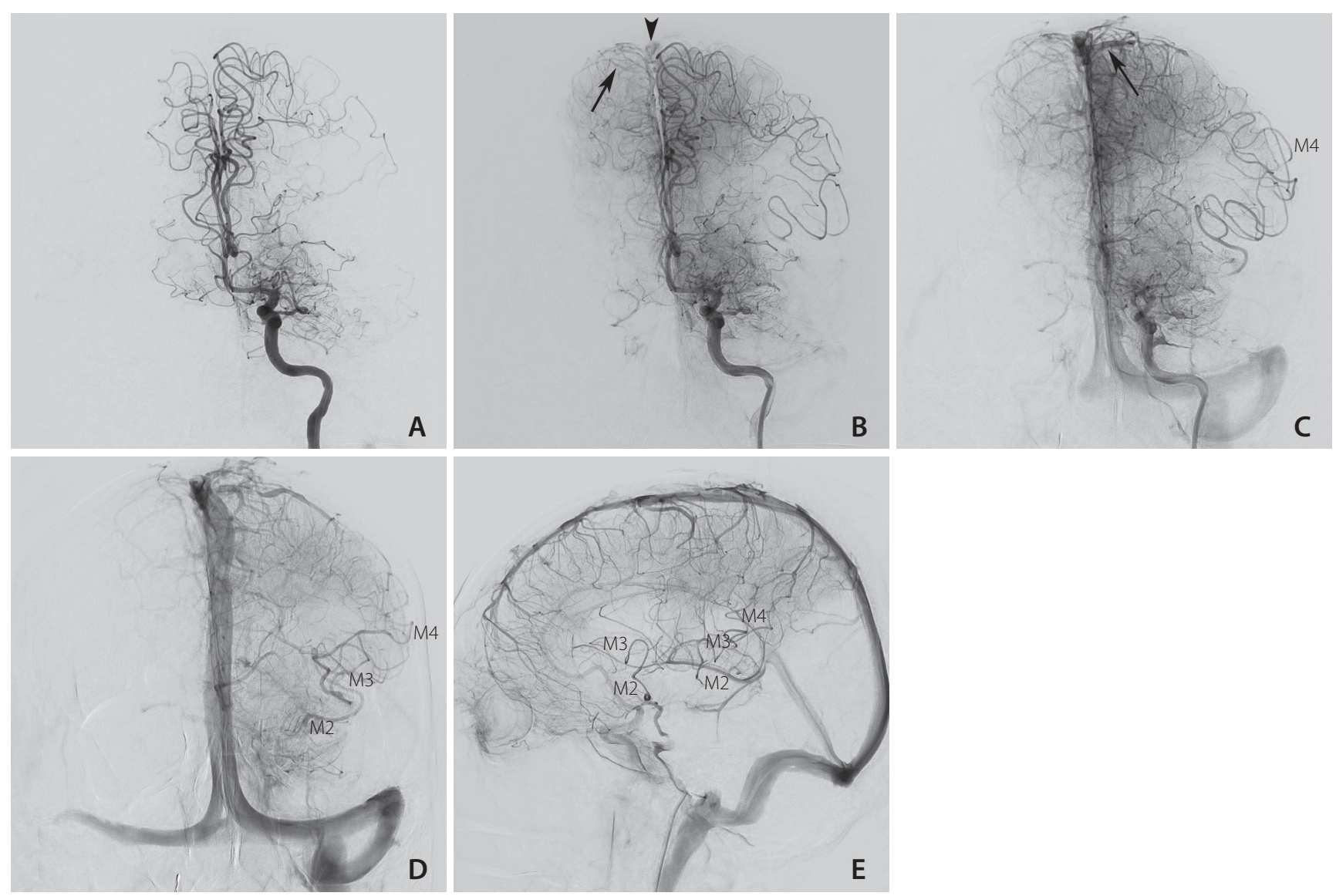

Fig. 1. Digital subtraction angiography in frontal projection (A-D) and lateral projection (E) after contrast injection in the left internal carotid artery showing the 4 seconds increase in arterio-venous transit time (AVTT) of the left anterior circulation compared to the right side. (A) The M1 segment of the middle cerebral artery (MCA) is occluded, both anterior cerebral arteries (ACA) are opacified at $t=6$ seconds. (B) The first cortical vein of the right ACA territory is visible at $\mathrm{t}=11$ seconds (arrow), with early contrast in the superior sagittal sinus (arrowhead). (C) The first cortical vein of the left ACA territory (arrow) is visible at $t=15$ seconds. (D, E) At the late arterial phase ( $t=21$ seconds), good collateral flow enables retrograde filling of the M4, M3 and M2 segments of the MCA.

we used ANOVA to compare per collateral grade the AVTT difference for the affected and unaffected hemisphere. Furthermore, the AVTT differences were tested for difference in age using a cut-off point of 75 years and two clinical parameters hypertension and diabetes mellitus using the independent samples t-test. Finally, the pre and post revascularization AVTT was measured to determine whether the delay was (partially) reversible with the paired t-test (both AVTT preand post revascularization were normally distributed).

\section{RESULTS}

A total of 364 patients subject to EVT were registered in our local 'stroke' database and underwent EVT. Only 41 of 364 LVO patients showed sufficient contrast flow across the
ACOM to allow AVTT determination on the contralateral side. Mean age for the selected patients was 67.8 years (range 38-88 years), 21 patients were female (51.2\%). AVTT for the affected side was 8.6 (standard deviation [SD] 2.4 seconds) and for the unaffected side 6.7 seconds (SD 2.1 seconds), representing a mean difference of 1.9 seconds (SD 1.6 seconds) with a range from 0 to 6 seconds. This difference was statistically significant $(P<0.001)$. A more than four second delay in AVTT between affected and unaffected side was seen in six patients. In nine patients, no difference in AVTT between affected and unaffected side was observed (Supplementary Table 1). When subdividing AVTT per collateral grade, only a trend towards a prolonged AVTT comparing the affected and unaffected side (Table 1) was observed with improved retrograde collateral circulation but no significance was reached. 
Table 1. Univariate analysis AVTT versus collateral grade

\begin{tabular}{|c|c|c|c|}
\hline \multirow[b]{2}{*}{ Collaterals } & \multirow{2}{*}{$\begin{array}{c}\text { Mean } \pm \text { standard } \\
\text { deviation }\end{array}$} & \multicolumn{2}{|c|}{ 95\% Confidence interva } \\
\hline & & $\begin{array}{l}\text { Lower } \\
\text { bound }\end{array}$ & $\begin{array}{l}\text { Upper } \\
\text { bound }\end{array}$ \\
\hline M2 segment (s) & $1.9 \pm 1.6$ & 1.3 & 2.6 \\
\hline M3 segment (s) & $1.7 \pm 1.1$ & 0.9 & 2.5 \\
\hline M4 segment (s) & $1.4 \pm 1.5$ & 0.4 & 2.3 \\
\hline
\end{tabular}

Dependent variable: affected AVTT ipsilateral minus contralateral side.

AVTT, Arterio Venous Transit Time.

Patients over 75 years of age showed a significant smaller delay in AVTT between affected and unaffected side ( $P=0.025)$ as compared to the younger age group. $A$ trend towards an increase in delay in AVTT between affected and unaffected side ( $P=0.23$ ) was seen in six patients with diabetes mellitus from 1.7 to 2.8 seconds (range $0-5$ seconds). Contrary a trend towards a decrease in delay in AVTT between affected and unaffected side was seen in 21 hypertensive patients $(P=0.23)$ from 2.1 to 1.7 seconds. None of the patients in this selected cohort had an internal carotid artery stenosis.

In 30 patients (73\%) TICl 2b-3 revascularization of the MCA was obtained allowing assessment of the AVTT in the affected hemisphere after treatment and subsequent comparison with the AVTT of the affected hemisphere before treatment. All control DSA's were performed within 10 minutes after revascularization. The mean post treatment AVTT on the affected side was 7.4 seconds (SD 2.1 seconds), which was significantly faster than the AVTT of 8.6 seconds as seen before treatment $(P=0.005)$. No signs of an underlying (atherosclerotic) stenosis were seen on the post revascularization DSA. Because of (partially) restored flow to the MCA territory after revascularization, crossflow through the ACOM to the contralateral hemisphere could not be reliably assessed.

\section{DISCUSSION}

In this study, significant reductions in perfusion AVTT were found during acute MCA occlusion in the ACA territory of the affected hemisphere compared to the contralateral non-affected side. After successful reopening of the occluded MCA, this reduction rapidly although partially reversed. We interpret these findings as a neurovascular 'steal effect', where collateral flow from the ACA to the MCA territory reduces the pressure head for ACA territory perfusion.

We postulate a novel concept in which a simple vascular network analysis (see Appendix 1) provides an explanation of the current findings. There are three crucial elements: first, better collateral connections between the anterior and medial perfusion area will support the area at risk, but at the same time steal more blood from the anterior circulation. While this is generally well understood, a second element is the resistance in the larger anterior arteries, proximal to the collaterals. After stroke onset, flow through these vessels increases strongly due to vasodilatation of leptomeningeal collaterals to the occluded MCA territory. This causes the perfusion pressure of the anterior circulation to drop. The effect on anterior flow depends on a third element; the auto-regulatory range of the anterior circulation. If flow reserve remains available, flow is maintained by microvascular vasodilation. If anterior perfusion pressure falls more strongly, flow reserve is exhausted and the anterior area becomes under-perfused, as reflected by the observed increase in AVTT in the affected hemisphere. Whether or not this occurs in individual patients, depends on the balance of these three elements. The appendix provides a straightforward equation for this balance. While the presented model may be oversimplifying, it should be pointed out that apart from autoregulation in the anterior circulation, no other vasoactive responses to the acute stroke event are needed to explain the current findings. The observed trend of an increase in delay of the AVTT in the affected ACA territory in relation to a better collateral grade supports the steal effect phenomenon. Statistical significance for this relation was not reached but is probably due to the relative small number of patients in each subgroup.

A quantitative comparison of the AVTT in this study with CT or MR perfusion data is complicated, as AVTT measures the transit of arterially injected contrast from the first opacification of the distal ICA to the first venous opacification. In perfusion imaging, the mean transit time (MTT) measures the time between arterial inflow and venous outflow but then on a parenchymal level. The limitation of MTT is that it is derived from the ratio of the area under the impulse response curve to its peak amplitude. ${ }^{9}$ Accurate calculation of this area requires that the signal intensity returns to baseline values during the scan acquisition, however in acute stroke patients with delayed transit times, this does not always occur. The most accurate estimation of a MTT penumbral threshold was calculated to be 5.3 seconds with a sensitivity 
of $88 \%$ and a specificity of $78 \% .^{10}$ The 1.7 second delay in AVTT of the anterior circulation due to steal is therefore not expected to cause large neurological damage in this area. Yet, these data do show that such steal is strong enough to cause exhaustion of autoregulation in this area. In diabetic patients, who have impaired autoregulation, the maximum delay in AVTT of five seconds as seen in this study is just below this penumbral threshold.11 Diabetic patients might be especially vulnerable for such hypoperfusion, with possible ischemic changes in the anterior circulation. ${ }^{12}$ In the present study the influence of hypertension on AVTT was equivocal. This might be explained by the fact that most of these patients received antihypertensive medication, which can restore autoregulation. ${ }^{13}$

The main limitation of this study is the dependence on a patent ACOM to visualize the contralateral circulation. A little more than 10 percent of patients showed enough ACOM cross flow to allow reliable AVTT measurements of the contralateral side. This might be attributed to a dominance of the contra-lateral A1, preventing overflow even when the ACOM is present and patent. With the advent of $\mathrm{CT}$ perfusion techniques it might be possible to measure the AVTT times per specific region of interest, including the contralateral ACA and MCA territory of the unaffected hemisphere. This might produce more robust data and conclusions. Another potential limitation might be the size of the ACOM that might influence crossflow to the contralateral side. However, even with a possible arterial inflow delay through the ACOM to the unaffected ACA, a more rapid passage of contrast was seen through the parenchyma into the draining veins.

\section{CONCLUSION}

An arterial steal phenomenon is present in stroke patients with proximal large vessel occlusion to augment collateral circulation to the MCA penumbral area. The presence of leptomeningeal collaterals is individually determined, and this should be taken in consideration when searching for means to improve cerebral blood flow during acute ischemic stroke due to large vessel occlusion.

\section{SUPPLEMENTARY MATERIALS}

Supplementary materials related to this article can be found online at https://doi.org/10.5469/neuroint.2020.00269.

\section{Fund}

None.

\section{Ethics Statement}

The study protocol and analysis of data was approved by the medical ethics committee of our institution; no informed consent was deemed necessary for this study by the medical ethics committee of our institution.

\section{Conflicts of Interest}

The authors have no conflicts to disclose.

\section{Author Contributions}

Concept and design: RB, OAB, HAM, and ETB. Analysis and interpretation: RB, JJW, and ETB. Data collection: RB, JJW, and $O A B$. Writing the article: RB, JJW, RVI, HAM, CBLMM, DV, and ETB. Critical revision of the article: RB, JJW, OAB, RVI, HAM, CBLMM, DV, and ETB. Final approval of the article: RB, JJW, OAB, RVI, HAM, CBLMM, DV, and ETB. Statistical analysis: JJW and ETB. Overall responsibility: RB.

\section{ORCID}

René van den Berg: https://orcid.org/0000-0002-8791-8791 Jenna J. Wildeman: https://orcid.org/0000-0001-9627-2200 Olvert A. Berkhemer: https://orcid.org/0000-0002-2212-6128 Rogier V. Immink: https://orcid.org/0000-0003-2427-6514 Henk A. Marquering: https://orcid.org/0000-0002-1414-6313 Charles B.L.M. Majoie: https://orcid.org/0000-0002-7600-9568 Dagmar Verbaan: https://orcid.org/0000-0002-9212-5470 Ed T. van Bavel: https://orcid.org/0000-0001-8654-986X

\section{REFERENCES}

1. Goyal M, Menon BK, van Zwam WH, Dippel DW, Mitchell PJ, Demchuk AM, et al.; HERMES collaborators. Endovascular thrombectomy after large-vessel ischaemic stroke: a meta-analysis of individual patient data from five randomised trials. Lancet 2016:387:1723-1731

2. Liebeskind DS. Collateral circulation. Stroke 2003;34:2279-2284

3. Bang OY, Saver JL, Buck BH, Alger JR, Starkman S, Ovbiagele B, et al.; UCLA Collateral Investigators. Impact of collateral flow on tissue fate in acute ischaemic stroke. J Neurol Neurosurg Psychiatry 2008;79:625-629 
4. Berkhemer $O A$, Jansen IG, Beumer D, Fransen PS, van den Berg LA, Yoo AJ, et al.; MR CLEAN Investigators. Collateral status on baseline computed tomographic angiography and intra-arterial treatment effect in patients with proximal anterior circulation stroke. Stroke 2016:47:768-776

5. Immink RV, van Montfrans GA, Stam J, Karemaker JM, Diamant $\mathrm{M}$, van Lieshout JJ. Dynamic cerebral autoregulation in acute lacunar and middle cerebral artery territory ischemic stroke. Stroke 2005;36:2595-2600

6. Aries MJ, Elting JW, De Keyser J, Kremer BP, Vroomen PC. Cerebral autoregulation in stroke: a review of transcranial Doppler studies. Stroke 2010;41:2697-2704

7. Berkhemer OA, Fransen PS, Beumer D, van den Berg LA, Lingsma HF, Yoo AJ, et al.; MR CLEAN Investigators. A randomized trial of intraarterial treatment for acute ischemic stroke. N Engl J Med 2015;372:11-20

8. Zaidat OO, Yoo AJ, Khatri P, Tomsick TA, von Kummer R, Saver JL, et al.; Cerebral Angiographic Revascularization Grading (CARG) Collaborators; STIR Revascularization working group; STIR Thrombolysis in Cerebral Infarction (TICI) Task Force. Recom- mendations on angiographic revascularization grading standards for acute ischemic stroke: a consensus statement. Stroke 2013;44:2650-2663

9. Butcher KS, Parsons M, MacGregor L, Barber PA, Chalk J, Bladin C, et al.; EPITHET Investigators. Refining the perfusion-diffusion mismatch hypothesis. Stroke 2005;36:1153-1159

10. Zaro-Weber O, Moeller-Hartmann W, Heiss WD, Sobesky J. MRI perfusion maps in acute stroke validated with 150-water positron emission tomography. Stroke 2010;41:443-449

11. Dandona P, James IM, Woollard ML, Newbury P, Beckett AG. Instability of cerebral blood-flow in insulin-dependent diabetics. Lancet 1979;2:1203-1205

12. Shuaib A, Butcher K, Mohammad AA, Saqqur M, Liebeskind DS. Collateral blood vessels in acute ischaemic stroke: a potential therapeutic target. Lancet Neurol 2011;10:909-921

13. Omura-Matsuoka E, Yagita Y, Sasaki T, Terasaki Y, Oyama N, Sugiyama $Y$, et al. Hypertension impairs leptomeningeal collateral growth after common carotid artery occlusion: restoration by antihypertensive treatment. J Neurosci Res 2011;89:108-116 


\section{Appendix 1. Network analysis of steal from the anterior circulation}

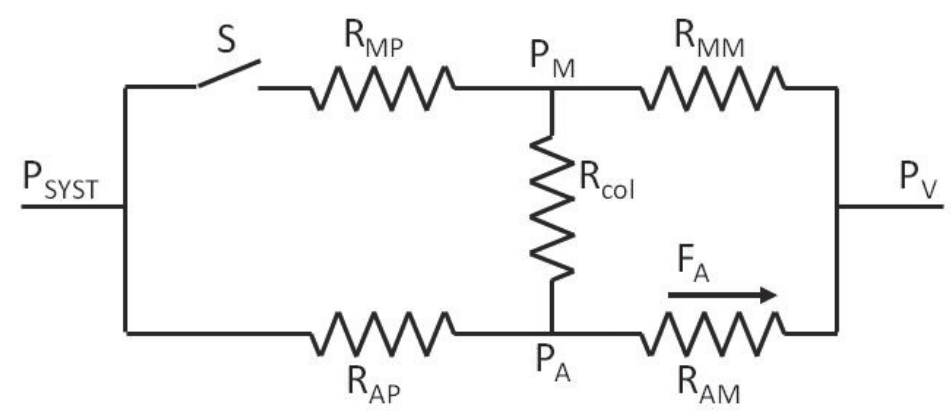

Fig. A1 provides the relevant resistances in the anterior and middle circulation after stroke. Opening of the switch $\mathrm{S}$ indicates the onset of stroke in the proximal MCA. The local pressures and flows before onset of stroke depend on the systemic pressure and venous back pressure (respectively $P_{S Y S T}$ and $P_{V}$ ), resistance in large anterior and MCA branches, proximal to the collaterals (respectively $R_{A P}$ and $R_{M P}$ ), the collateral resistance $R_{C O L}$, and the microvascular resistances in both the anterior and MCA perfusion areas (respectively $R_{A M}$ and $R_{M M}$ ). After onset of stroke, $R_{M P}$ becomes irrelevant but all other resistances together determine the driving pressure $\left(P_{A}\right)$ for flow through the anterior microcirculation $\left(F_{A}\right)$. Ignoring a possible small collateral flow before the onset of stroke, and taking $P_{V}=0$, anterior microvascular flow $\left(F_{A}\right)$ before and after onset of stroke equals:

Before stroke onset:

$$
F_{A}=P_{S Y S T} \cdot \frac{1}{R_{A P}+R_{A M}}
$$

After stroke onset:

$$
F_{A}=P_{S Y S T} \cdot \frac{R_{M M}+R_{C O L}}{\left(R_{M M}+R_{C O L}+R_{A M}\right) \cdot R_{A P}+\left(R_{M M}+R_{C O L}\right) \cdot R_{A M}}
$$

The anterior microvascular resistance is subject to autoregulation. It is well known that a reduction of systemic pressure causes vasodilation in order to maintain perfusion. Likewise, in this setting, a sudden drop in anterior driving pressure $\left(P_{A}\right)$ at onset of stroke will be compensated by a reduction of $R_{A M}$. Whether this is sufficient to maintain anterior perfusion at the autoregulatory level depends on the balance of resistances and the reserve in the anterior circulation. The anterior microvascular vasodilator reserve (MVR) can be defined as

$$
M V R \stackrel{\text { def }}{=} R_{A M}^{r e g} /_{R_{A M}^{d i l}}=G_{A M}^{d i l} / G_{A M}^{r e g}
$$

Where $R_{A M}^{d i l}$ is the minimal anterior microvascular resistance, under full vasodilation, and $R_{A M}^{r e g}$ the resistance under normal autoregulation, prior to stroke onset. Conductance $\mathrm{G}$ is the reciprocal of resistance.

Anterior flow then will be maintained within the autoregulatory range if

$$
M V R \geq 1+\frac{R_{A P}}{R_{M M}+R_{C O L}}
$$

A few numerical cases illustrate this: If the resistance in the large anterior arteries, $R_{A p_{1}}$ is negligible, no vasodilator reserve is needed to maintain anterior perfusion at the autoregulary level $(M V R=1)$. In this case, $P_{A}$ equals $P_{S Y S T}$ and the collateral flow is not imposing a load on the anterior circulation at all. Also in the absence of collaterals, $M V R=1$ is sufficient but in this case obviously the anterior and medial circulation are fully separated and there is no collateral flow. If $R_{A P}$ equals the resistance of the collaterals and medial microcirculation $\left(R_{C O L}+R_{M M}\right), M V R$ needs to be at least 2 in order to maintain anterior flow at the autoregulatory level during middle cerebral artery occlusion. 